\title{
Proceso de cuidado nutricional y desnutrición asociada a la enfermedad: ¿negligencia o injusticia?
}

\author{
Nutritional care process and disease-related malnutrition: \\ negligence or injustice? \\ Processo de cuidado nutricional e desnutrição associada à doença: \\ negligência ou injustiça?
}

Mónica López-Talavera**

Recibido: 18 de mayo de 2021. Aceptado para publicación: 4 de agosto 2021

https://doi.org/10.35454/rncm.v4supl1.298

\section{Resumen}

La malnutrición asociada a la enfermedad es una entidad clínica multifactorial que representa un problema sostenido a lo largo del tiempo, con implicaciones graves para el paciente y para los sistemas de salud. Además de los factores de riesgo que presenta el paciente como su entorno económico, sociocultural y estado clínico, también el factor de mala praxis del personal de salud influye en que la malnutrición asociada a la enfermedad no se detecte ni se trate y se mantenga.

La reflexión que se hace en este escrito parte de los principios de la ética biomédica de no maleficencia y justicia, ya que propone que la negligencia y la injusticia son factores agravantes de la malnutrición asociada a la enfermedad. Estas cuestiones, aunque pueden privar al paciente de ejercer su derecho humano al cuidado nutricional, están presentes por desconocimiento y falta de educación del personal de salud respecto a la importancia de la nutrición clínica y del proceso de cuidado nutricional.

La propuesta generada es que la educación en diferentes niveles, entendiéndose también como capacitación y cultura institucional, puede ser un área de oportunidad para minimizar el problema de la vulnerabilidad nutricional, respetando, defendiendo y manteniendo la dignidad humana con el cuidado nutricional mediante el estudio de la ética y la ética biomédica para incluir los cuatro principios bioéticos a la práctica clínica diaria.

Palabras clave: malnutrición, cuidado nutricional, derechos humanos, Declaración de Cartagena, bioética, nutrición clínica.
Abstract

Disease-associated malnutrition is a multifactorial clinical entity that represents a problem sustained over time with serious implications for the patient and for health systems. In addition to patientrelated risk factors such as the economic and sociocultural environment and clinical status, malpractice by healthcare personnel also plays a role in the failure to detect and treat disease-related malnutrition and its persistence.

The reflection made in this paper is based on the principles of biomedical ethics of non-maleficence and justice. It proposes that negligence and injustice are compounding factors of disease-related malnutrition. These are issues that, besides depriving patients of their ability to exercise their human right to nutritional care, they exist because of lack of knowledge and lack of healthcare staff education regarding the importance of clinical nutrition and nutritional care.

The proposal is that education at different levels, understood also as training and institutional culture, can be an area of opportunity to minimize the problem of nutritional vulnerability, by respecting, defending and maintaining human dignity with nutritional care through the study of ethics and biomedical ethics to include the four bioethical principles in daily clinical practice.

Keywords: Malnutrition; Nutritional Care; Human Rights; Cartagena Declaration; Bioethics; Clinical Nutrition.

\section{Resumo}

A desnutrição associada à doença é uma entidade clínica multifatorial que representa um problema que se mantém ao longo do tempo com graves implicações para o paciente e para os sistemas de saúde. Além dos fatores de risco apresentados pelo paciente como sua situação econômica, sociocultural e clínica; O fator de práxis inadequada do pessoal de saúde também influencia o fato de que a desnutrição associada à doença não possa ser detectada, tratada e mantida.

A reflexão feita neste escrito se baseia nos princípios da ética biomédica da não maleficência e da justiça, propondo que a negligência e a injustiça são agravantes da desnutrição associada à doença, questões que, embora possam privar o paciente de exercer seus direitos humanos ao cuidado nutricional, estão presentes devido ao desconhecimento e falta de educação do pessoal de saúde quanto à importância da nutrição clínica e do processo de cuidado nutricional.

A proposta gerada é que a partir da educação em diferentes níveis, também entendida como formação e cultura institucional, possa ser um espaço de oportunidade para minimizar o problema da vulnerabilidade nutricional, respeitando, defendendo e mantendo a dignidade humana com os cuidados nutricionais, por meio do estudo da ética e a ética biomédica para incluir os quatro princípios bioéticos na prática clínica diária.

Palavras-chave: desnutrição, cuidado nutricional, direitos humanos, Declaração de Cartagena, bioética, nutrição clínica. 
' Consultorio Diaitetik, Nutrición Clínica Especializada; Universidad Autónoma del Estado de México. Toluca, México.
*Correspondencia: Mónica López Talavera. mlt88rm@gmail.com

\section{INTRODUCCIÓN}

En Latinoamérica, los registros actualizados de desnutrición asociada a la enfermedad (DAE) reportaron una prevalencia del 2,6 \%-73,2 \% ${ }^{(1)}$, misma que no ha presentado cambios desde su primer registro estadístico publicado en $1974^{(2)}$. La DAE es una entidad multifactorial en la que se ha reconocido el papel de factores socioeconómicos y patológicos del paciente para su instauración y progresión ${ }^{(3)}$, pero también se debe tener presente la influencia de la práctica clínica deficiente de cuidado nutricional y conocimiento respecto a la importancia de la nutrición clínica, como se ha identificado en estudios anteriores ${ }^{(1,2,4)}$. Se ha observado que el proceso de cuidado nutricional es aplicado en menos del $50 \%$ de los pacientes, de los cuales el 11,9\% es identificado con DAE y el $17,6 \%$ es identificado con riesgo de DAE; en menos del $50 \%$ se monitoriza la ingesta alimentaria (aunque haya antecedentes de disminución de esta y de pérdida de peso no intencional), en menos del $46 \%$ hay intervención de personal de nutrición, y menos del $41 \%$ de expedientes tienen datos de riesgo nutricional o $\mathrm{DAE}^{(5)}$.

A nivel mundial, la prescripción de terapia nutricional a pacientes en riesgo se encuentra en $29 \%$, mientras que en Latinoamérica esta prescripción se ha estimado en $32 \%$. Las barreras que pueden estar generando esta problemática aún no han sido definidas y deben comenzar a ser identificadas y reflexionadas para obtener propuestas de solución ${ }^{(6)}$. Al igual que los factores causantes y agravantes, también se conocen cuáles son las consecuencias que impactan de forma negativa al pronóstico del paciente por el aumento de riesgo de reingresos, reintervenciones, morbimortalidad, días de estancia hospitalaria y $\operatorname{costos}^{(1,4)}$. Pero más allá de estos efectos se debe cuestionar el impacto del conjunto de factores de riesgo, perpetuantes y orgánicos, en la integridad y dignidad humana: ¿qué sucede con la persona que la padece?

De acuerdo con el quinto principio de la Declaración de Cartagena, se reconoce desde una postura ética al individuo con riesgo o DAE como una persona vulnerable, ya que la afectación también se encuentra en su autonomía, su capacidad de alimentarse y pone en riesgo su vida; estas situaciones requieren la intervención de los profesionales de nutrición clínica ${ }^{(7)}$. Reconocer al paciente como una persona vulnerable destaca la necesidad de tener el cuidado del otro para mantener intacta su dignidad humana, a pesar del compromiso de su integridad y autonomía por el mismo proceso que representa la DAE, y las implicaciones de dependencia de un cuidador $^{(8)}$. Sin embargo, al tener la detección y registro de las fallas en la atención al paciente en cuanto a la solicitud y oportunidades de intervención de la nutrición clínica para ejercer y proteger el cuidado nutricional, como proceso y derecho humano emergente, es necesario realizar una reflexión a partir de dos principios de ética biomédica: la no maleficencia y la justicia, pues con ellos se puede intentar encontrar una justificación ante las deficiencias de la práctica clínica referentes al proceso del cuidado nutricional que agravan la vulnerabilidad del paciente desde el punto de vista nutricional, así como el impacto en la integridad humana de acuerdo con el enfoque de Derechos Humanos.

\section{DISCUSIÓN}

\section{No maleficencia: primero, no dañar}

El principio de no maleficencia se centra en la abstención de una acción que pueda causar daño, entendiéndose este último como el impedimento, por actos intencionados o no, de que se cumplan los intereses de una de las partes sin violar sus derechos ${ }^{(9)}$.

Para evitar y disminuir el riesgo de daño es fundamental ser conscientes del cuidado debido, el cual no está presente cuando se expone al paciente a un riesgo inaceptable o de forma inintencionada, o cuando se actúa sin cumplir los criterios morales que exige la profesión al ser adquiridos durante la formación; esto resulta en un daño por desconocimiento ${ }^{(9)}$.

De acuerdo con la evidencia, la problemática que representa la DAE está agravada por la práctica con desconocimiento o la ausencia de la misma, que desde la ética biomédica puede ser entendida como negligencia. Esto es así porque se ha demostrado que no hay una preparación adecuada del personal médico ni de enfermería en cuanto a la educación en nutrición clínica, siendo reconocida esa deficiencia por ellos mismos ${ }^{(10,11)}$. 
El no realizar tamizaje a todos los pacientes durante las primeras $24-48$ horas de su ingreso hospitalario, no solicitar interconsulta a nutrición clínica, y no tener la disponibilidad de recursos y la organización institucional para establecer el cuidado nutricional pueden ser acciones no intencionadas por desconocimiento, lo que se traduce en un problema por negligencia y causa la afectación en la integridad humana; esto aumenta la vulnerabilidad y atenta contra el derecho humano al cuidado nutricional.

\section{Justicia: oportunidad justa e igualitaria}

La justicia en la ética biomédica se refiere al merecimiento de los beneficios que por derecho a cada quien le corresponden, entendiendo que hay injusticia cuando actos erróneos no permiten obtenerlos ${ }^{(9)}$.

$\mathrm{Su}$ aplicación es con la justicia distributiva, siendo posible la distribución de derechos y responsabilidades en la sociedad para cubrir las necesidades fundamentales evitando causar daño. Esto se apoya por las teorías igualitaristas que versan sobre la distribución igualitaria de los recursos a partir de la organización institucional y social para que todos tengan el acceso a las necesidades básicas ${ }^{(9)}$.

De esta manera, se puede asegurar la oportunidad justa para que se disponga de servicios de atención a la salud cuando el paciente esté en desventaja (enfermedad) que no pueda evitar por sí mismo. Esta atención debe ser en proporción a la gravedad de la enfermedad, resaltando su carácter equitativo, el cual está relacionado con niveles organizacionales para la asignación y administración de recursos ${ }^{(9)}$.

Al no brindar cuidado nutricional al paciente que se encuentre en desventaja, es decir, con riesgo o padeciendo la DAE, además de negligencia, podría catalogarse como una injusticia. En la actualidad existe evidencia de que la DAE puede ser una entidad clínica tratada con intervenciones costoefectivas, tanto para el paciente como para las instituciones, cuyo ahorro proyectado por paciente es hasta $41,5 \%$ menos con terapia nutricional temprana, así como $23,8 \%$ menos en costos de hospitalización y $66,4 \%$ menos en costos de readmisiones. Esto representa un ahorro anual en salud pública de hasta $32,1 \%{ }^{(12)}$.

Es inaceptable que a pesar de eso no se dé prioridad o incluso sea ignorado como opción terapéutica el cuidado nutricional, cuestión que actualmente no depende del paciente ${ }^{(13)}$, pues los recursos para implementar el cuidado nutricional dependen del conocimiento de los médicos tratantes y del personal de enfermería, de la organización y administración de las instituciones, y del gobierno ${ }^{(5)}$.

En el mismo tenor, si se sabe que las necesidades básicas de alimentación, vida digna y salud que son reconocidas como Derechos Humanos no son aseguradas durante la atención médica del paciente, se estaría dejando de cumplir con el deber de buscar la oportunidad justa para que se ejerzan y se protejan los derechos humanos, en los que también se reconoce el cuidado nutricional $^{(14)}$.

\section{Cuidado nutricional como derecho humano}

Con la Declaración de Cartagena. Declaración Internacional sobre el Derecho al Cuidado Nutricional y la Lucha contra la Malnutrición los Derechos Humanos a la salud, la vida digna y la alimentación se unen en el derecho humano emergente del cuidado nutricional, y se lucha porque se asegure para todas las personas en condiciones de vulnerabilidad nutricional ${ }^{(14)}$. Los antecedentes de esta propuesta se encuentran en la Declaración de Cancún, documento que reconoce el derecho humano a la alimentación y soporte nutricional, enfatizando en el deber de tener un abordaje nutricional óptimo y oportuno en todo paciente hospitalizado ${ }^{(15)}$.

Diez años después el derecho humano reconocido en 2008 evoluciona al cuidado nutricional, mismo que también incluye la protección contra el hambre y el derecho a la alimentación con la terapia nutricional, pero se fundamenta y orienta en la protección y el respeto de la dignidad del paciente mediante el proceso de cuidado nutricional con el enfoque de Derechos Humanos ${ }^{(16,17)}$.

El derecho al cuidado nutricional se distingue porque se busca asegurar que las personas sean alimentadas, ya sea porque no tienen la capacidad propia para hacerlo o porque su condición clínica requiere que la alimentación sea administrada por vías artificiales, es decir, que se encuentran vulnerables ${ }^{(16)}$.

La propuesta del derecho al cuidado nutricional tiene un enfoque particular en el paciente enfermo hospitalizado, pues la misma entidad clínica puede generar una disminución de la ingesta alimentaria, aunque se tengan los alimentos disponibles, por renuencia o por imposibilidad dependiendo del cuadro clínico; o porque se encuentre en la etapa final de la vida y la alimentación ya no sea posible, o el cuadro clínico impida la alimentación ${ }^{(17)}$.

Además, se intenta que el problema de la DAE y el hambre en el ámbito clínico se contemplen en la agenda 
internacional, ya que son atentados no intencionales (negligencia) contra la dignidad humana (si no se permite el acceso al derecho humano hay injusticia) que no se deben permitir ${ }^{(18)}$.

La responsabilidad para ejercer el cuidado nutricional es compartida por tres actores: el paciente, el personal de salud y el Estado. El deber del Estado es administrar los recursos para que se pueda tener un abordaje de cuidado nutricional óptimo, oportuno y de calidad permitiendo el acceso al derecho humano; el deber y derecho del paciente es reclamar su cuidado nutricional; y el deber del nutricionista y demás personal de salud de asegurar que se cumpla todo el proceso de cuidado nutricional ${ }^{(16)}$ para salvaguardar ese derecho. De esta manera se logrará respetar la dignidad humana, pues se ejerce la autonomía y con las indicaciones se respeta la dimensión humana y biopsicosocial del paciente ${ }^{(18)}$.

Sin embargo, al tener evidencia que refleja una falta de cuidado nutricional óptimo, oportuno y seguro, posiblemente por negligencia como se analiza en apartados anteriores, también se puede entender como un atentado contra los derechos humanos de la persona en vulnerabilidad nutricional ${ }^{(17,18)}$, lo que refleja una injusticia.

\section{Educar: posible solución}

La DAE es una entidad compleja, pero puede ser prevenida y tratada con mayor eficacia si se contempla la necesidad de educar en todos los niveles posibles respecto al impacto de la nutrición clínica para encontrar la solución, especialmente si se conjunta con la reflexión ética y de ética biomédica para la práctica clínica.

Si el análisis para la solución parte del cuidado nutricional como derecho humano, se corre el riesgo de comenzar a visualizar el problema como un agravio a la dignidad humana, lo cual posiciona al personal de salud en un ámbito deshumanizado en el que la máxima de no dañar no se tomaría como parte de su ejercicio, razón de ser profesional y de vocación ${ }^{(19)}$.

$\mathrm{Y}$ aunque pudiera ser válido este punto de partida para justificar los actos de negligencia e injusticia que se involucran en la perpetuación y gravedad de la DAE, no puede considerarse válido desde el fundamento de que el personal de salud tiene la vocación especial de servicio con las máximas de no dañar y hacer el bien al consultante, siempre buscando su recuperación mediante la oportunidad justa y con conocimiento de causa ${ }^{(19)}$.

En contraste, si el análisis se realiza contemplando el desconocimiento o ignorancia del proceso de cuidado nutricional como punto de partida, la negligencia e injusticia que se muestran en la práctica deficiente pueden ser justificadas, ya que, al no saber la consecuencia de los actos, no puede clasificarse como un atentado contra la dignidad y derechos humanos, pues no se hace con el propósito de dañar ${ }^{(9)}$.

Con actos basados en el desconocimiento puede ser comprensible por qué no se tienen los recursos, la organización ni la educación necesaria, tanto en el personal como en las instituciones de salud, de la importancia del cuidado nutricional para la dignidad humana y su impacto en la administración del sistema de salud.

La evidencia muestra que, a pesar de la formación deficiente en materia de nutrición clínica de todo el personal de salud, existe el interés para saber más acerca de esta ${ }^{(10,19)}$ mediante la colaboración para la práctica en el ámbito clínico nutricional ${ }^{(20)}$, ya que la calidad de la terapia nutricional también es cuestión de la identificación y monitorización de los actores involucrados en el equipo multidisciplinario, especialmente personal médico y de enfermería ${ }^{(11)}$.

Como se menciona en apartados anteriores, la exigencia moral de la profesión se obtiene durante la formación ${ }^{(9)}$, especialmente con una sensibilidad humana desarrollada con el estudio de la ética y la ética biomédica, donde se enmarcan y comprenden los principios bioéticos que deben regir el ejercicio profesional.

Esta exigencia es la misma que no se puede dejar de lado una vez que es comprendida, porque se transforma en la guía de la forma de ser profesional y humana; sin embargo, cuando ni siquiera es conocida o concientizada no se tendrán las herramientas necesarias y suficientes para poder cumplir el compromiso ético y moral.

$\mathrm{Si}$ la ética se une con la propuesta para mejorar la educación en materia de nutrición clínica en el personal de salud, los tres rubros principales que darán conocimientos para las competencias en la nutrición básica, nutrición aplicada y nutrición clínica permitirán que el personal médico tenga la capacidad de: reconocer la importancia de la nutrición y su impacto en el ser humano; orientar a la población en cuanto a estilo de vida saludable; y saber, como profesionales de la salud, en qué momento es necesario referir al paciente con un especialista de nutrición clínica ${ }^{(21)}, y$ así poder salvaguardar el derecho al cuidado nutricional.

El paciente también debe ser educado respecto a la importancia del cuidado nutricional, pues educarle es empoderarle y promover que sea libre y autónomo para la toma decisiones, al mismo tiempo que es responsable de su autocuidado para la prevención o el tratamiento de la DAE, e incluso pueda exigir la atención de nutrición clí- 
nica si conoce los factores de riesgo nutricional y el impacto que puede existir con una intervención a tiempo ${ }^{(22)}$.

De esta manera, dentro los principios bioéticos contemplados dentro de la educación y promoción del cuidado nutricional se podrían reforzar los principios 3, 8, 9 y 10 de la Declaración de Cartagena, que contemplan la importancia de la educación y proponen su desarrollo en el paciente, el personal, las instituciones y el sistema de salud mediante capacitaciones, redes de servicios de nutrición clínica, investigación, cursos de pregrado y posgrado, organización y legislación para que el cuidado nutricional pueda ser realidad como un derecho humano ${ }^{(14)}$.

\section{CONCLUSIONES}

La identificación y tratamiento deficiente de la DAE es un problema al que se enfrenta la nutrición clínica desde hace mucho tiempo, debido al desconocimiento de la importancia que representa identificar la vulnerabilidad nutricional de los pacientes que la padecen o se encuentran con riesgo de padecerla.

Esto es así porque los actos no intencionados, sin buscar el mal del paciente, se realizan por una falta de adquisición de criterios y deberes morales como consecuencia de una educación deficiente sobre la ética, el cuidado nutricional y el impacto de la nutrición clínica.

Esta manera de actuar puede verse desde la ética biomédica como negligente e injusta, ya que el cuidado nutricional es inexistente o deficiente por falta de educación; esto al mismo tiempo tiene graves consecuencias éticas, morales, humanas y económicas, pues los actos erróneos no permiten que se ejerza y asegure el derecho humano al cuidado nutricional, cayendo en la injusticia y en un posible atentado contra la dignidad humana y los derechos humanos.

Como se mencionó, la responsabilidad para hacer del cuidado nutricional una realidad es compartida, ya que todos tienen un deber que cumplir. El Estado debe asegurar que se cuenten con los recursos e infraestructura; las instituciones deben desarrollar una cultura organizacional que incluya al cuidado nutricional como prioridad; el personal de salud debe actuar para garantizar el proceso de cuidado nutricional; y el paciente debe exigirlo por derecho.

Al ser la DAE una entidad compleja porque su desarrollo y solución no se encuentra en un solo frente, la ética en conjunto con la educación en materia de nutrición es el pilar básico para que los diversos actores puedan contribuir a que se proteja y ejerza el cuidado nutricional. De esta manera, las exigencias morales podrán cumplirse por tener la formación, capacitación, organización y conciencia del impacto del cuidado nutricional en el respeto de la dignidad humana, y podrán establecerse estrategias más eficaces para combatir la DAE al tiempo que se disminuye el riesgo de caer en una práctica de negligencia e injusticia para con el paciente y sus derechos humanos.

\section{PUNTOS CLAVE}

- La malnutrición asociada a la enfermedad es una entidad clínica compleja, por lo que es importante reconocer los factores de riesgo que se encuentran en la práctica del personal de salud.

- De acuerdo con la ética biomédica, la mala práctica por desconocimiento refleja negligencia e injusticia, pues se priva al paciente de poder beneficiarse de su derecho al cuidado nutricional.

- Para disminuir el desconocimiento del problema se debe compartir la responsabilidad de la educación en todos los niveles del sistema de salud, desde el político y legislativo hasta el de la formación de personal de salud y la cultura institucional.

- Buscar asegurar el cuidado nutricional como derecho humano también implica la educación para empoderar al paciente y que exija su derecho en cualquier nivel de atención a la salud.

- Se debe aprovechar la ventana de oportunidad que representa la Declaración de Cartagena. Declaración Internacional sobre el Derecho al Cuidado Nutricional y la Lucha contra la Malnutrición en cuanto a la difusión del concepto de malnutrición relacionada a la enfermedad, y el interés del personal de salud por conocer más del ejercicio e importancia de la nutrición clínica.

\section{Declaración de autoría}

MLT se encargó de la revisión y análisis de referencias, así como a la redacción de todo el artículo.

\section{Conflicto de intereses}

La autora declara no tener conflicto de intereses.

\section{Financiación}

El presente estudio no tuvo financiación. 


\section{Referencias bibliográficas}

1. Correia MITD, Perman MI, Waitzberg DL. Hospital malnutrition in Latin America: A systematic review. Clin Nutr. 2017;36(4):958-67. doi: 10.1016/j.clnu.2016.06.025

2. Butterworth CE. Skeletton in the hospital closet. Nutrition Today. 1974;9(2):4-8.

3. Cederholm T, Barazzoni R, Austin P, Ballmer P, Biolo G, Bischoff SC, et. al. ESPEN guidelines on definitions and terminology of clinical nutrition. Clin Nutr. 2017;36(1):49-64. doi: 10.1016/j.clnu.2016.09.004

4. Correia MITD, Campos ACL, ELAN Cooperative Study. Prevalence of hospital malnutrition in Latin America: the multicenter ELAN study. Nutrition. 2003;19(10):823-5. doi: 10.1016/s0899-9007(03)00168-0

5. Hiesmayr M, Tarantino S, Moick S, Laviano A, Sulz I, Mouhieddine $\mathrm{M}$, et al. Hospital Malnutrition, a Call for Political Action: A Public Health and NutritionDay Perspective. J Clin Med. 2019;8(12):2048. doi: 10.3390/ jcm8122048

6. Cardenas D, Bermúdez C, Pérez A, Diaz G, Cortes LY, Contreras CP, et al. Nutritional risk is associated with an increase of in-hospital mortality and a reduction of being discharged home: Results of the 2009-2015 nutritionDay survey. Clin Nutr ESPEN. 2020;38:138-45. doi: 10.1016/j. clnesp.2020.05.014

7. Cardenas BD, Echeverri S, Puentes M, Pérez A, López L y Bermúdez C. Los 13 Principios de la Declaración de Cartagena. Rev Nutr Clin Metab. 2019;2(Supl.1):24-41. doi: 10.35454/rncm.v2supl1.035

8. Cardenas D, Echeverri S. Aspectos éticos de la Declaración de Cartagena. Rev Nutr Clin Metab. 2019;2(Supl.1):56-62. doi:

9. Beauchamp TL, Childress JF. Principios de ética biomédica. Madrid: Masson S. A; 1999.

10. Cardenas D, Díaz G, Cadavid J, Lipovestky F, Canicoba M, Sánchez P, et al. Nutrition in medical education in Latin America: Results of a cross-sectional survey. JPEN J Parenter Enteral Nutr. 2021. doi: 10.1002/jpen.2107

11. Papier I, Lachter J, Hyams G, Chermesh I. Nurse's perceptions of barriers to optimal nutritional therapy for hospitalized patients. Clin Nutr ESPEN. 2017;22:92-96. doi: 10.1016/j. clnesp.2017.08.002

12. Sulo S, Vargas J, Gomez G, Misas JD, Serralde-Zúñiga AE, Correia MITD. Hospital nutrition care informs potential costsavings for healthcare: A budget impact analysis. Clin Nutr ESPEN. 2021;42:195-200. doi: 10.1016/j.clnesp.2021.01.041

13. Barazzoni R. Nutrición y Nutrición Clínica como derechos humanos. Rev Nutr Clin Metab. 2019;2(Supl.1):12-13. doi: 10.35454/rncm.v2supl1.028

14. Cardenas D, Bermúdez C, Echeverri S, Pérez A, Puentes M, López L, et al. Declaración de Cartagena. Declaración Internacional sobre el Derecho al Cuidado Nutricional y la Lucha contra la Malnutrición. Nutr Hosp. 2019;36(4):97480. doi: 10.20960/nh.02701

15. Castillo PJC de la C, Figueredo GR, Dugloszewski C, Diaz RJASR, Spolidoro NJV, Matos A, et al. Declaración de Cancún: declaración internacional de Cancún sobre el derecho a la nutrición en los hospitales. Nutr Hosp. 2008;23(5):413-7.

16. Cardenas BD, Echeverri S, Bermúdez C. Del derecho a la alimentación al derecho al cuidado nutricional. Rev Nutr Clin Metab. 2019;2(Supl 1):42-48. doi:

17. Cardenas D, Correia MITD, Ochoa JB, Hardy G, RodriguezVentimilla D, Bermúdez CE, et al. Clinical Nutrition and Human Rights. An International Position Paper. Nutr Clin Pract. 2021;36(3):534-44. doi: 10.1002/ncp.10667

18. Cardenas D. Nutrición clínica y el enfoque basado en derechos humanos. Rev Nutr Clin Metab. 2019;2(Supl.1):49-55. doi:

19. Laín Entrelago P. La medicina hipocrática. Madrid: Ed. Revista de Occidente; 1970.

20. Crowley J, Ball L, Hiddink GJ. Nutrition in medical education: a systematic review. Lancet Planet Health. 2019;3(9):e379-e389. doi: 10.1016/S25425196(19)30171-8

21. Cuerda C, Muscaritoli M, Donini LM, Baqué P, Barazzoni R, Gaudio et al. Nutrition education in medical schools (NEMS). An ESPEN position paper. Clin Nutr. 2019;38(3):969-74. doi: 10.1016/j.clnu.2019.02.001

22. Correia MITD. Patient Empowerment on the Fight Against Malnutrition. JPEN J Parenter Enteral Nutr. 2018;42(4):67274. doi: $10.1002 /$ jpen 